\title{
Balkanologie
}

Balkanologie Revue d'études pluridisciplinaires

Vol. XIV, $\mathrm{n}^{\circ}$ 1-2 | 2012

Volume XIV Numéro 1-2

\section{Les Tsiganes comme autochtones. Le cas des Roms Gabori - Roumanie}

\section{Martin Olivera}

\section{(2) OpenEdition}

\section{Journals}

Édition électronique

URL : http://journals.openedition.org/balkanologie/2340

DOI : 10.4000/balkanologie.2340

ISSN : 1965-0582

Éditeur

Association française d'études sur les Balkans (Afebalk)

Référence électronique

Martin Olivera, "Les Tsiganes comme autochtones. Le cas des Roms Gabori - Roumanie », Balkanologie [En ligne], Vol. XIV, n 1-2 | 2012, mis en ligne le 26 janvier 2013, consulté le 17 décembre 2020. URL : http://journals.openedition.org/balkanologie/2340 ; DOI : https://doi.org/10.4000/ balkanologie.2340

Ce document a été généré automatiquement le 17 décembre 2020.

(C) Tous droits réservés 


\title{
Les Tsiganes comme autochtones. Le cas des Roms Gabori - Roumanie
}

\author{
Martin Olivera
}

1 «Les Tsiganes sont originaires d'Inde »: voilà généralement la première chose qu'apprend le curieux s'intéressant à la question. Sans même nécessiter de plus amples développements, l'information revêt un fort pouvoir analytique : elle permettrait d'expliquer à la fois leur étrangeté (culturelle), leur marginalité (sociale) mais également le rejet dont ils font l'objet de la part des populations européennes se définissant comme autochtones.

2 L'origine identifiée grâce aux travaux de linguistique autorise d'autre part à penser la présence tsigane selon cette puissante rhétorique nationale, réifiant les identités collectives et structurant le champ symbolique européen depuis deux siècles : chaque " peuple » s'identifie à une origine géographique donnée, à une langue particulière, à un folklore etc. ${ }^{1}$ Ce n'est à ce titre pas un hasard si les Tsiganes sont devenus objet du discours savant, puis médiatique et populaire, à l'heure de la construction des idéologies nationales en Europe : en français le terme générique Tsigane, opposé aux diverses autres appellations populaires (Bohémien, Romanichel, Nomade, etc.), est attesté en $1826^{2}$. Ces groupes furent ainsi associés dès l'origine aux discours nationaux chaque pays a élaboré son Tsigane sur un canevas commun, l'étranger de l'intérieur tout en en étant exclus, définitivement, par un mécanisme radical : le déni d'histoire.

3 Les nations européennes se prévalent en effet d'une autochtonie plus ou moins mythique dans laquelle se perdent leurs racines. Celtes ou Gaulois, Ibères, Daces, Pictes et Étrusques fondent la légitimité naturelle des peuples sur leur territoire. On note néanmoins que l'affirmation nationale se construit en s'émancipant radicalement de ces lointains aïeux, par l'Histoire et la Civilisation : les Gaulois sont nécessaires aux Français mais, socialement et culturellement, les Français d'aujourd'hui ne sont en rien des Gaulois, et ne se pensent pas comme tels. "Ce sont nos ancêtres, mais nous ne sommes pas leurs héritiers ", tel est le paradoxe de l'autochtonie moderne ${ }^{3}$. Or, cette mutation essentielle, par laquelle une nation justifie dans un même mouvement sa légitimité territoriale et sa vertu auto-civilisatrice, semble déniée aux Tsiganes. Ceux-ci 
seraient bel et bien les descendants directs de leurs aïeux. "Société froide" de l'Europe, ils n'auraient somme toute qu'assez peu évolué depuis leur départ de l'Hindoustan, il y a plus de mille ans, au point qu'il n'apparaît pas nécessaire d'employer un autre terme pour les distinguer de ceux que les tsiganologues ont identifié comme leurs lointains ancêtres. «Les Tsiganes (ou les Rroms) ${ }^{4}$ viennent du sous-continent indien ", dit-on, en employant un présent éternel. Ils rejoignent par-là ces sociétés primitives, censément ignorées de l'Histoire jusqu'à leur rencontre avec l'homme blanc ${ }^{5}$... C'est ainsi que l'historiographie des Tsiganes correspond avant tout à celle de l'attitude des institutions à leur égard et à la manière dont ils subissent la politique des Gajé. Comment, dès lors, ne pas avoir l'image d'une "population victime ", tenue en marge, sans ancrage et ballottée au hasard des aléas secouant les sociétés européennes ${ }^{6}$ ?

Pourtant, depuis maintenant plus de vingt ans, nombre d'auteurs démontrent qu'il convient de prendre en compte le passé circonstancié et l'insertion locale des différentes communautés - bien plutôt qu'un lointain exotisme fondateur - si l'on souhaite comprendre leurs réalités socioculturelles ${ }^{7}$. Je souhaite ici poursuivre dans cette voie, au travers d'un exemple roumain particulièrement éloquent : les Gabori de Transylvanie. Ce faisant, on saisira l'intérêt qu'il peut y avoir à regarder les communautés tsiganes comme des populations autochtones, d'un genre particulier.

5 L'exemple gabor démontre que les Tsiganes sont parfois (souvent ?) mieux ancrés dans un territoire et son histoire que les populations les entourant. Ces Roms témoignent du caractère actif de cet ancrage et de leur insertion locale. Si, comme toute société humaine, ils n'ont pas un contrôle total sur leur devenir collectif, ils n'en deviennent pas pour autant de simples victimes passives des bouleversements sociaux et historiques. Encore faut-il, pour s'en rendre compte, quitter les perspectives globalisantes et prêter attention aux discours des intéressés, dans leur contexte local et régional.

Les données et analyses ici présentées sont issues d'une enquête ethnographique extensive menée entre 1999 et 2007 (sur près de 30 mois cumulés) auprès de familles gabori, essentiellement dans les régions de Deva et Târgu-Mureș. Chez ces Roms, comme dans d'autres sociétés tsiganes (les auteurs sont nombreux à en témoigner), le terme "enquête" peut néanmoins paraître peu approprié: il s'agit bien plutôt d'engranger un maximum d'observations en se laissant mener par ses hôtes dans leur quotidien - pour s'associer progressivement à celui-ci - sans nécessairement poser beaucoup de questions... Bien souvent, les réponses à ces dernières constituent par ailleurs les données les moins utiles à l'analyse, les Roms étant bien trop habitués à contenter le Gajo dans sa curiosité. Non qu'ils aient des choses à cacher, tels les secrets garants de leur «tsiganité", mais, plus fondamentalement, en raison de leur incompréhension totale vis-à-vis de notre intérêt pour l'identité comme objet : ils ne tiennent, entre Gabori, quasiment aucun discours sur eux-mêmes en tant que groupe. Ces Roms passent en revanche beaucoup de temps à évaluer, nouer, dénouer et renouer les liens qui unissent, de près ou de loin, les familles et individus de la société gabor. L'appartenance collective est dès lors une expérience quotidienne, aussi évidente que difficilement exprimable, bien plutôt qu'un sentiment identitaire catégoriel ${ }^{8}$.

7 Peut-être plus encore que d'autres Autres, les Tsiganes rappellent par là au savant que la réalité sociale n'est faite que d'histoires personnelles. L'ethnographe peut dès lors, à titre individuel, trouver sa place dans la sociabilité rom, s'il consent à donner de lui- 
même en nouant des relations personnelles approfondies et renonce, au moins pour le temps du séjour chez ses hôtes, à ses objets et autres raisonnements désincarnés. À cet égard, plutôt que d' "observation participante ", je caractériserais pour ma part mon travail de "participation observée ", à rebours, lorsqu'il fallut ordonner un discours anthropologique au regard de mes expériences de terrain. La quasi-totalité des données mobilisées dans l'analyse relèvent ainsi du registre de l'anecdote, vécue ou rapportée, mettant en scène des personnages. Pour reprendre les mots de M. Stewart, «I can now honestly say that the bulk of my data comes from direct observation and questioning only in context. With one or two exceptions I found impossible to use general questioning productively. This is a desperately frustrating position to be in, but I believe my restraint gradually won me the respect and confidence of Rom I got to know well ».

Précisons enfin que l'omniprésence du terme «Gabor » dans les lignes à venir ne vise qu'à clarifier le propos et ne reflète pas l'usage des intéressés, ceux-ci réservant l'emploi de l'ethnonyme aux relations avec les Gajé, afin de se distinguer clairement des autres Tsiganes. Dans l'entre-soi gabor, en romanès, il n'est pas nécessaire de préciser : en disant simplement «Nous, les Roms » (Ame, Roma), ceux-ci ne font pas référence à une minorité ethnique transnationale (ni même nationale) mais, de manière parfaitement évidente à leurs yeux, aux seuls semblables définis par le champ des relations de parenté (filiation et alliance), c'est-à-dire, ici, à ceux qui se disent et se reconnaissent Gabori. Et ce « Nous » apparaît indissociable du territoire dans lequel il s'exprime.

1.

\section{Une « nation » de Roms transylvains}

Depuis quelques années, les Gabori apparaissent au grand public comme une communauté tsigane éminemment «traditionnelle » : ils parlent romanès, portent des vêtements spécifiques qui les distinguent tout autant des autres Tsiganes que des Gajé, pratiquent pour beaucoup un "métier traditionnel» (la ferblanterie-zinguerie, en particulier la pose de gouttières), revendiquent une stricte endogamie, etc. Nonobstant cette image d' «authenticité tsigane », l'identité originale de ces Roms doit tout, dans ses raisons comme dans sa forme, à leur autochtonie ou, si l'on préfère, à la profonde intégration de cette communauté à son environnement.

On rencontre aujourd'hui des Gabori dans toute la Transylvanie, et même au-delà : aux confins actuels de leur zone d'expansion, certaines familles sont installées à Bucarest, Iaşi, Constanţa ou Craiova. Leur nombre est estimé entre 3000 et 5000 individus. Tous se reconnaissent comme membres d'une même «nation » (naŢia Gaborilor), ayant pour origine un territoire situé dans le département de Mureş (fig. 1). En effet, tous les groupes familiaux gabori proviennent d'un réseau de communes rurales situé aux alentours de la ville de Târgu-Mureş - Marosvasarhély en hongrois. Certains Roms ont quitté ce territoire originel depuis fort longtemps, parfois plus de 40 ans. Ils ne manquent néanmoins pas de dire, lorsqu'ils s'y rendent pour un mariage, pour y visiter des parents ou honorer la tombe d'un défunt : «Je rentre à la maison » (žav khere).

Le département de Mureş (judeţul Mureş) est majoritairement peuplé de magyarophones. Avec ses voisins de Harghita et Covasna, il correspond approximativement aux anciens territoires sicules de Transylvanie. Les Sicules (Secuii en roumain, Szekely en hongrois) forment une population de langue hongroise, installée 
depuis le Haut Moyen Âge au centre de l'arc carpatique - on y reviendra (fig. 3). Il convient toutefois de donner dès à présent quelques éléments de l'histoire et de la géographie roumaine. Car si les Roumains (autrefois désignés comme Valaques) y sont majoritaires, la Transylvanie accueille diverses minorités. Leur longue histoire commune a forgé l'identité particulière de la région: localement majoritaires, trois de ces minorités (Hongrois, Saxons - terme désignant les groupes de langue allemande - et Sicules) ont longtemps dominé politiquement la principauté.

Le rattachement de la Transylvanie aux principautés de Moldavie et Valachie en 1919 (Union de la "Grande Roumanie »- România Mare) consacra pour les nationalistes roumains un réajustement "naturel" de l'Histoire et la fin d'une trop longue domination hongroise. Jusqu'alors, les principautés moldo-valaque, d'une part, et la Transylvanie ${ }^{10} \mathrm{~d}$ 'autre part, constituaient deux ensembles marqués par des influences distinctes. Les régions occidentales entretinrent des rapports étroits avec la couronne hongroise, puis avec l'empire habsbourgeois (jusqu'à y être absorbées), tandis que les principautés moldaves et valaques, davantage influencées par le monde slave, restèrent sous domination ottomane du XV ${ }^{\text {ème }}$ au XIX ${ }^{\text {ème }}$ siècle.

13 Sous sa forme actuelle, le pays a ainsi moins d'un siècle d'existence, et cinquante années de communisme centralisateur n'ont pas arasé les identités locales. Celui qui souhaite comprendre la Roumanie actuelle et, partant, comprendre les Roms de Roumanie, doit dès lors prendre en considération les forts régionalismes qui s'expriment aujourd'hui encore au quotidien.

14 En ce sens, nombre de Transylvains (qu'ils soient hongrois, roms ou roumains) regardent leurs concitoyens de Moldavie et de Valachie (en particulier les olténiens) avec un œil condescendant: ces derniers seraient en grande partie des "arriérés " (înapoiaŢi), habitants de régions orientales « défavorisées ", tandis que la Transylvanie, plus riche et historiquement tournée vers l'Ouest, s'affirme " moderne » et « civilisée ». 
Figure 1. Roumanie : grandes régions historiques et départements actuels

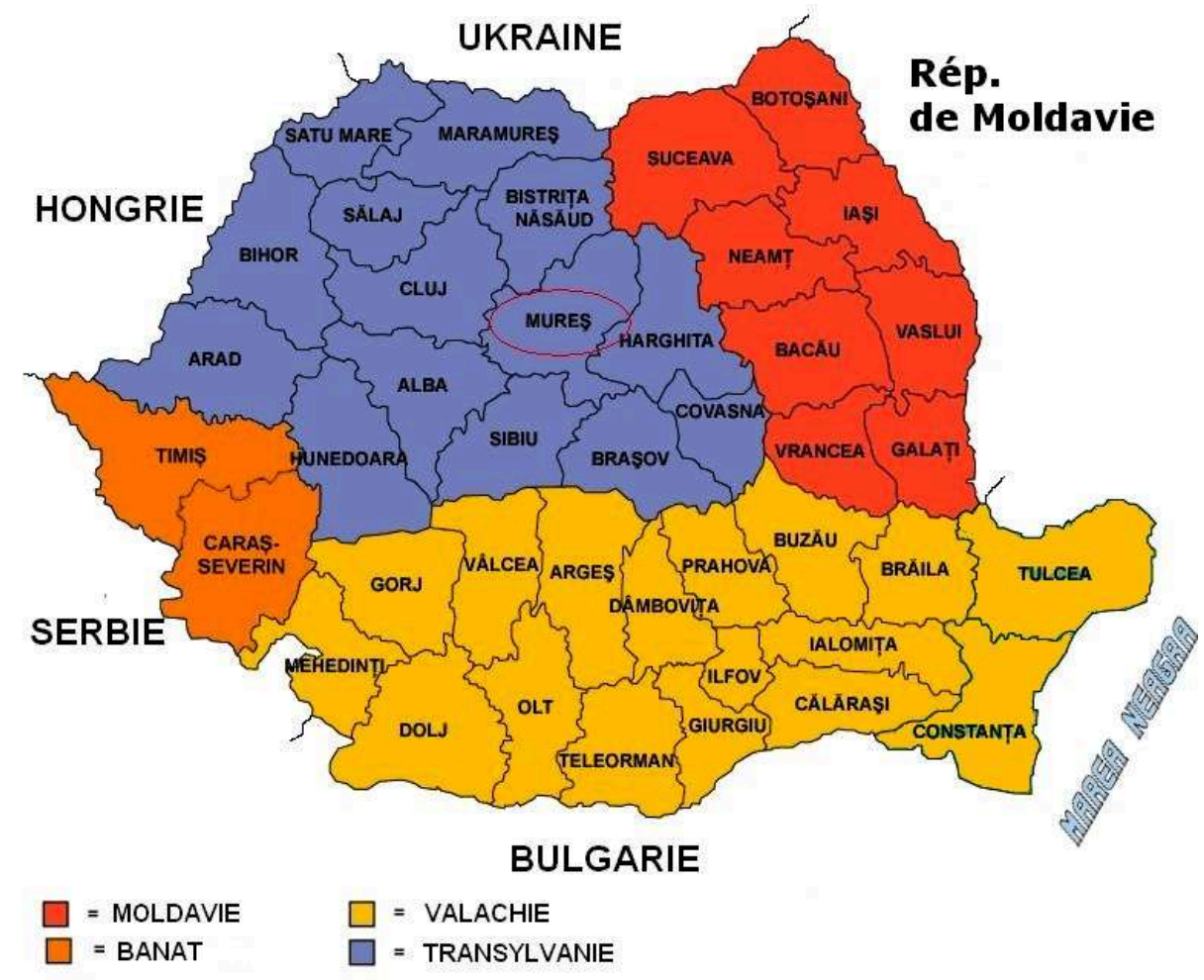

Ce regard sur l'Autre se décline à l'intérieur même des grandes régions historiques. En Transylvanie, nombres de stéréotypes circulent ainsi sur les habitants de telle ou telle contrée (terroir, ville ou département) ${ }^{11}$. Ils varient bien évidemment selon l'appartenance du locuteur.

Bien loin de se tenir à l'écart de ces jeux de miroir identitaires, les Gabori y participent pleinement : à leurs yeux les Gajé ne sont pas tous identiques, loin de là, de même que les autres Tsiganes sont qualifiés, appréciés ou dépréciés selon leur lieu d'origine. À ce titre, ils estiment être bien plus proches des Roumains et Hongrois de Cluj ou de SatuMare que des Tsiganes d'Alexandria (département de Teleorman - Munténie) ou de Bârlad (département de Vaslui - Moldavie). La manière dont se formule et s'affirme l'identité gabor apparaît ainsi indissociable de la « géographie symbolique » roumaine : en tant que Tsiganes hongrois, de par leur origine mureşienne, ces Roms jouissent dans la région d'une image de sérieux et de droiture morale. Leur magyaritude les occidentalise et, ce faisant, les civilise. De même, l'étiquette «Roms de la Mureş » fait d'eux un groupe dont on identifie l'origine : ils ne viennent pas de nulle part et, par là même, rassurent les Gajé.

Les Gabori présentent donc aux observateurs l'image d'un "clan tsigane » (neam Tiganesc) «noble et respectable» (rajkane the patjivale Roma disent-ils en romanès), porteur d'une tradition ancestrale positive ${ }^{12}$. Contrairement à nombre d'autres Roms, ils possèdent un ethnonyme univoque, leurs vêtements sont à la fois typiques, soignés et traditionnels, ils semblent former une communauté soudée, etc. Tous ces signes sont pertinents dans le cadre d'une logique identitaire fondée sur l'idéologie nationale réifiante. Les Gabori font dès lors figure d'exception dans le paysage tsigane, tel que le peignent les représentations nationales populaires : leur « identité » n'est pas définie de manière uniquement négative. 

leurs semblables n'a rien d'anodin ${ }^{13}$. En Transylvanie, le mot est historiquement et symboliquement lié à l'idée de noblesse et de respectabilité : ce sont les trois « nations nobiliaires" (hongroise, saxonne et sicule) qui dominèrent politiquement la principauté durant toute l'époque Moderne, tandis que la plèbe valaque ne formait par définition pas une "nation $»^{14}$. En s'affirmant comme naŢia, nécessairement "noble et respectable ", les Gabori se distinguent de la masse indistincte des Ţigani (décombres symboliques de l'âge féodal et de la servitude) ${ }^{15}$ en même temps qu'ils s'établissent à égalité des Gajé « civilisés », porteurs d'une longue et estimable histoire locale.

1.

\section{L'émergence des Gabori, « clan tsigane traditionnel »}

19 On peut toutefois constater que cette affirmation collective gabor, calquée sur le modèle «national », est tout à fait récente. Ce n'est qu'après la Révolution de 1989 que ces Roms s'affirment publiquement comme une communauté particulière, très visible dans le paysage transylvain. De ce point de vue, l'étude du «costume traditionnel » gabor, et de son évolution récente, paraît révélatrice.

Notons par exemple que les chapeaux masculins n'ont eu de cesse de s'élargir depuis la chute du régime de Ceausescu, cette croissance s'étant même accélérée à la fin des années 1990 et au début des années 2000, tel que j'ai moi-même pu l'observer. Un chapelier d'Alba-Iulia, auprès duquel s'approvisionne une bonne partie des Gabori, s'est ainsi progressivement spécialisé dans la confection de ces larges couvre-chefs, ceux-ci constituant aujourd'hui la majeure partie de son chiffre d'affaire (en 2007, un tel chapeau coûtait entre 150 et 250 euros).

De même, les vêtements féminins se sont faits plus colorés, ornementés et, finalement, "exotiques», au cours des deux décennies passées. L'étude des photographies familiales montre que, jusqu'aux années 1980, hommes et femmes portaient un costume plus terne et moins éloigné de celui de leurs voisins hongrois : chapeaux, longues moustaches, gilet et pantalons larges pour les hommes, fichu, chemisier brodé, jupe (plissée et colorée les jours de fêtes) et tablier pour les femmes, étaient déjà les éléments du vêtement gabor, mais également ceux du costume de tout un chacun en milieu rural transylvain - du moins était-ce le cas jusque dans les années 1950-1960. Aujourd'hui encore, les Romnia (femmes roms) font confectionner leurs jupes très plissées (il faut en moyenne huit mètres de tissu par jupe) par de vieilles couturières hongroises, même si ces dernières sont de moins en moins nombreuses et que certaines Romnia assurent depuis quelques années, à leur tour, la perpétuation de ce savoir-faire. 
Costumes gabori
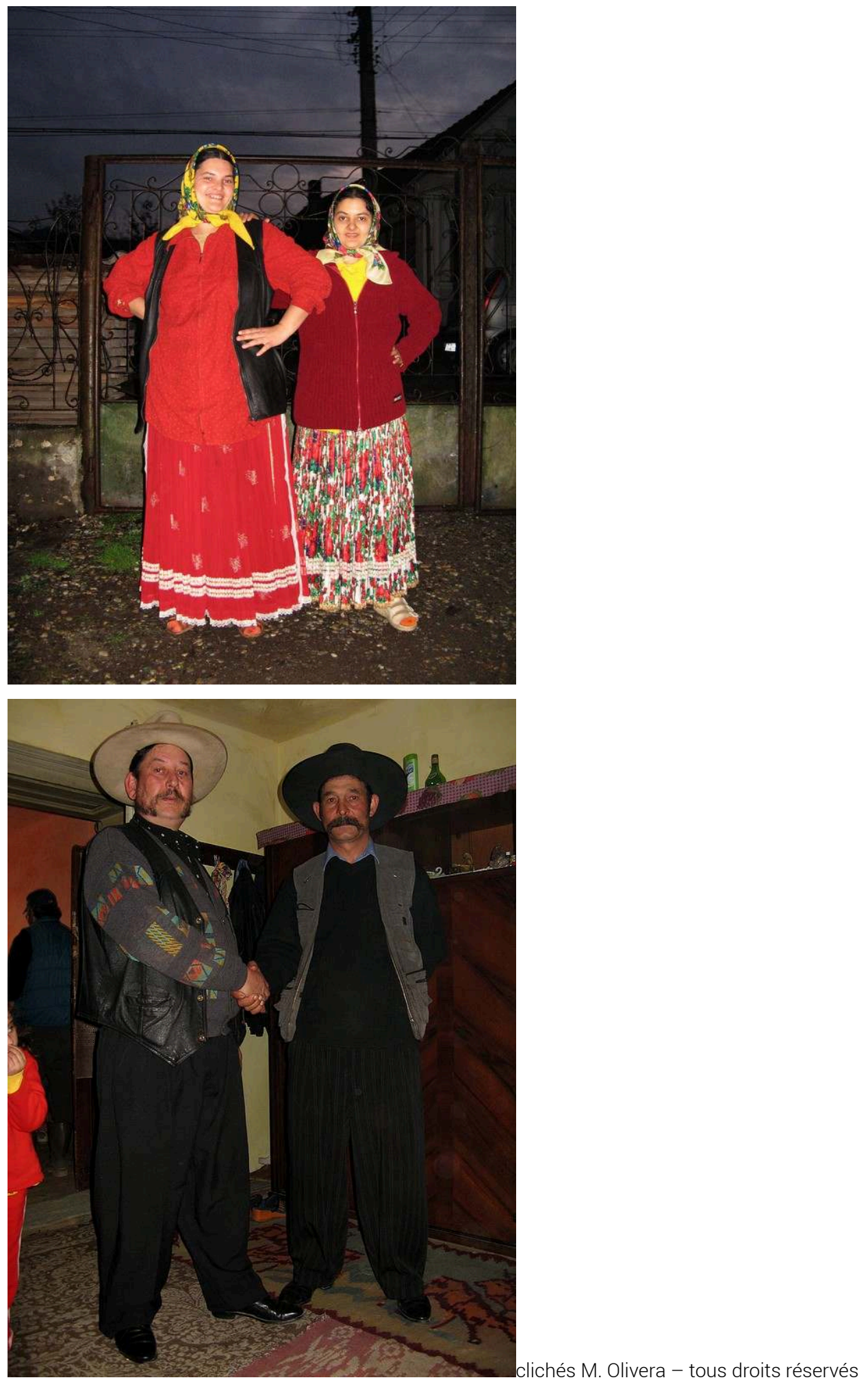

Les vêtements «traditionnels » et " authentiquement tsiganes » des Gabori (personne, Rom ou Gajo, ne songerait à les rapprocher du patrimoine vestimentaire local) n'ont ainsi rien d'étranger au territoire dans lequel ceux-ci évoluent, de leur point de vue, depuis toujours. 

discrète, sur la scène publique ? Plusieurs éléments peuvent être avancés. Tous sont, là encore, indissociables d'un contexte régional et national dont les Gabori sont membres (actifs) à part entière.

(ans tout d'abord que la visibilité gabor se développe alors que ceux-ci se sont éparpillés dans toute la Roumanie, bien au-delà de leurs villages d'origine, à partir de la fin des années $1950^{16}$. L'idée de communauté elle-même n'est bien entendu pas apparue ex-nihilo au cours des années 1960-1970, puisque la mémoire familiale des vị̧i (pseudolignages structurant la parenté gabor, $c f$. plus bas) remonte jusqu'aux premières décennies du XX ${ }^{\text {ème }}$ siècle. Ce qui semble nouveau au tournant des années 1980 est une formalisation publique du discours communautaire.

Avant d'aller plus loin, un retour sur quelques exemples de parcours familiaux au cours des années 1960-1980 permettront de se faire une meilleure idée de la manière dont les Gabori ont pu se disperser au cours du régime communiste, loin de leur territoire d'origine, tout en maintenant une forte structure communautaire.

1.

\section{0-1980 : itinéraires familiaux sous le communisme}

Pişta, cinquante ans aujourd'hui, a un arrière-grand-oncle maternel ayant, d'après ses dires, combattu en France dans les tranchées au sein de l'armée austro-hongroise. Il avait également un grand-oncle paternel qui, pour sa part, a passé plusieurs années comme prisonnier de guerre chez les Russes durant la Seconde Guerre. Le fils de ce dernier possède une vieille photographie encadrée de son père en uniforme hongrois. Celle-ci trône en bonne place dans la pièce principale. Pișta sait que, dans les années 1950, des Roms ont commencé à partir s'installer dans des grandes villes de Transylvanie, notamment à Oradea (Nagyvàrad en hongrois, Bari varadi en romanès) et à Cluj. Mais en ce qui concerne sa propre famille élargie, les Roms demeuraient à l'époque dans leurs villages, pratiquant différents métiers pour gagner leur vie: forgerons et rétameurs pour certains, mais aussi briquetiers, journaliers, etc.

Le premier proche à avoir quitté la région de Tîrgu-Mureş fut l'un des frères de sa mère, par le biais de l'armée : l'oncle fit son service militaire au début des années 1960 dans la région de Timişoara, à Buziaş. Certains disent qu'il a même été officier, en tout cas il se faisait appeler "le colonel» (o colonel). Une fois retourné dans le civil, ce dernier resta à Buziaş et s'y installa avec femme et enfants. Il avait désormais de nombreuses connaissances (prinženimo) «bien placées » dans la ville et la région. Déjà ferblantier à l'époque, il se forma à la couverture-zinguerie, auprès d'autres Roms mais aussi de Gajé. Deux de ses frères vinrent le rejoindre là-bas, où « le colonel » leur apprit le travail de la tôle zinguée. Ils travaillèrent quelques temps avec lui, puis s'en allèrent de leur côté : l'un à une trentaine de kilomètres de là, à Făget, l'autre plus au sud, dans le département de Caraş-Severin. Les trois frères n'en continuèrent pas moins d'assurer de fréquents allers-retours entre la région de Tîrgu-Mureş (pour visiter les proches, y entretenir leur maison, etc.) et leur lieu de travail-résidence dans le Banat.

Pour leur part, c'est à la fin des années 1960 que Pişta et sa famille quittèrent leur petit village mureşien : le gigantesque chantier d'une centrale thermoélectrique commença en 1967 près de Deva. Manquant localement de main-d'œuvre, les autorités recrutèrent des bras, dans la région de Tîrgu-Mureş notamment. Dans le village de Pişta ils furent 
nombreux à s'engager : six familles roms déménagèrent, toutes apparentées, soit en tout une trentaine de personnes. D'après les Roms, les conditions salariales étaient alors très intéressantes, d'autant qu'ils manquaient de travail dans leur village d'origine. Ils ne furent pas les seuls à venir du département de Mureş pour travailler à la construction de la centrale, d'autres Tुigani (non-Gabori ceux-là) mais aussi des Hongrois et quelques Roumains les accompagnèrent.

Nos Roms pratiquaient ainsi toutes sortes de métiers sur l'immense chantier: manœuvre, conducteur de machine, pelleteur, ferblantier, maçon... En dehors des heures de travail, ceux qui connaissaient le métier pratiquaient également à leur compte le rétamage et la zinguerie dans les villages avoisinants. Si le père de Pişta savait travailler le fer et le cuivre, il n'était pas ferblantier-zingueur. Son frère aîné avait pour sa part appris le métier d'un autre Rom. Pişta partit ainsi habiter deux années, dans le département de Harghita, où son frère s'était établi quelques années auparavant, afin d'apprendre la couverture-zinguerie. Puis il revint à Deva, s'engagea à la termo et se mit parallèlement à exercer ce métier à son compte.

30 Tous ces Roms parlent des années 1970-1980 comme d'une belle époque : ils étaient logés dans des baraquements neufs construits par l'État et bien payés, avaient de bonnes relations avec le voisinage, etc. Et puis quand la centrale fut mise en service en 1980, certains s'y engagèrent tandis que d'autres rentrèrent chez eux, ou bien partirent travailler ailleurs, grâce aux relations développées au cours des années passées dans ce nouveau territoire.

31 D'autres Gabori, d'un autre village de Mureş, s'étaient installés à Deva dès le début des années 1960, quelques années avant que Pişta et ses proches ne viennent eux-mêmes dans la région. Ils y furent rejoints dans le courant des années 1980 par des Roms apparentés (venant de la région de Braşov où ils s'étaient établis au début des années 1970). Deux de ceux-là prirent pour épouses les sœurs de Pişta. Tous les Gabori présents aujourd'hui à Deva et dans sa proche région, soit une vingtaine de maisons, sont ainsi originaires de villages autour de Tîrgu-Mureş, bien que certains les aient quittés depuis près de 40 ans.

32 Tels qu'en témoignent les récits et parcours gabori, l'éparpillement entraîne ainsi l'éloignement d'ensembles familiaux, qui ne se côtoient désormais qu'occasionnellement (notamment pour des mariages, enterrements et autres fêtes collectives), mais aussi leur recomposition. Il faut dès lors pouvoir être reconnu, par ses semblables comme par ces nouveaux Gajé qui voient arriver des Tsiganes se voulant «positivement différents» ( $c f$. ci-dessus). Le costume gabor permet cette reconnaissance univoque et maintient l'évidence communautaire par-delà l'éclatement spatial. Où qu'il aille, un Gabor est certain de reconnaître un autre Gabor lorsqu'il le croise. Eux seuls portent un tel costume, permettant d'identifier a priori un semblable (sur le quai d'une gare, à une station service, dans un marché...) et, en lui adressant la parole, de vérifier qu'il s'agit bien d'un membre de la même "nation »: au fil de la discussion, les divers liens de parenté unissant les deux interlocuteurs seront progressivement démêlés, alors même qu'ils ne s'étaient jamais rencontrés auparavant.

33 L'éparpillement gabor apparaît directement lié à une meilleure intégration de leur terroir originel à la Roumanie communiste : les familles quittent alors progressivement leurs villages de Mureş pour des villes et des régions jugées plus riches, embauchant de la main-d'œuvre (lancement de grands chantiers, développement de centres urbains), 
plus aisément accessibles grâce à l'amélioration des réseaux routiers et ferrés, etc. C'est aussi à cette époque que le métier de ferblantier-zingueur se répand de proche en proche parmi ces Roms, jusqu'à devenir « traditionnel ».

Voici donc des "Tsiganes du village", vivant essentiellement de l'agriculture de subsistance dans un territoire restreint, qui deviennent en une génération des «Roms traditionnels » très visibles, maniant le marteau, bien plus mobiles que leurs parents et grands-parents, et ce sous un régime censé avoir œuvré à l'«assimilation » et à la " sédentarisation » des Roms ${ }^{17}$. De l'utilité de circonstancier et de localiser observations et analyses lorsque l'on tient un discours sur des Tsiganes: en Roumanie comme ailleurs, les réalités sont diverses et l'expérience des uns ne vaut pas nécessairement pour les autres.

Un autre élément permet de comprendre le «choix» gabor de s'afficher ostensiblement comme une communauté rom particulière, plus encore à partir des années 1990.

Les années qui suivent la chute de Ceausescu mettent la figure du Tsigane sur le devant de la scène publique. Si le régime socialiste niait l'existence de minorités ethniques en Roumanie, pour mieux développer l'image d'un seul et même peuple de travailleurs, les années dites « de transition » font du " problème tsigane » un symptôme révélateur des graves problèmes nationaux. Impossible dès lors pour les Roms d'être invisibles. En s'affichant comme Tsiganes, soit, mais naturellement et positivement différents des autres Tsiganes, les Gabori semblent avoir joué une carte féconde. La première chose qu'ils précisent à tout nouvel interlocuteur, plus encore lorsque celui-ci semble étranger, équivaut ainsi à une mise en garde, jouant sur la connivence : « Ah mais nous on est des Tsiganes, oui !, mais pas comme ceux-là, qui boivent, qui sont voleurs et dangereux! Non, nous on est des Tsiganes nobles, respectables ! On est connu partout pour ça. Etc. » Et de fait, je ne compte plus le nombre de Roumains ou de Hongrois qui, tout en déplorant les problèmes causés par "les Tsiganes ", me déclaraient à propos de leurs voisins ou amis gabor : "Ah oui mais eux c'est différent, il n'y a qu'à les regarder. »

La manière dont se formulait l'identité collective gabor au début du XX ${ }^{\text {ème }}$ siècle devait être bien différente de sa forme actuelle. Était-il alors seulement question de Gabori ? Rien n'est moins sûr... À ma connaissance, l'ethnonyme "Gabor " n'apparaît dans aucun document avant les années 1990. Les études sur les Roms de Roumanie étaient certes moins nombreuses sous le régime communiste mais il existe des travaux approfondis menés dans les années 1920-1930 : les Gabori, ces Tsiganes «traditionnels authentiques" n'y apparaissent pas identifiés en tant que groupe spécifique. Les intéressés devaient simplement dire, comme bien des Roms: «Nos Roms » - Amare Roma. Et l'on constate que l'ethnonyme, élément pourtant essentiel dans l'affirmation d'une identité ancestrale et immuable, s'adapte aux conditions du marché identitaire local, lui-même indissociable des mutations sociopolitiques.

Preuve est faite que ces Roms ne sont pas restés en marge de l'histoire, mais y ont été pris et y ont participé, à leur manière. L'émergence progressive de cette "nation des Gabori » aux yeux de tous peut ainsi être lue comme une conséquence tout autant qu'un facteur de l'intégration de ces familles à leur environnement régional et, plus largement, national.

Il y a néanmoins plus à dire de l'usage de l'autochtonie et du "traditionalisme " gabor que l'instrumentalisation dont ils peuvent faire l'objet vis-à-vis des Autres, dans une logique désormais bien connue de jeu sur l'ethnicité ${ }^{18}$ et de réinvention perpétuelle de 
la «tradition $»^{19}$. Si ces Roms mettent en avant les notions de "noblesse» et d'autochtonie pour se définir, c'est qu'ils estiment être réellement " nobles » (rajkane Roma) et autochtones. Pour comprendre comment ces deux dimensions ne sont que les deux faces d'une seule et même qualité qui, aux yeux des Gabori, les distinguent tout autant des autres Tsiganes que des Gajé, un retour vers leur terre d'origine s'impose.

1.

\section{« D'où es-tu ? »-Katar san ? : vivre l'autochtonie}

Lorsque deux Gabori ne se connaissant pas se rencontrent, soit parce qu'ils accompagnent chacun d'autres Roms qui se connaissent, soit parce qu'ils se sont reconnus comme semblables dans un lieu public (grâce aux vêtements en particulier), les deux questions qui suivent les salutations d'usage sont invariables : «D'où es-tu? ? (Katar san?) et «De qui es-tu?» (Kako san?). Si la première peut parfois être posée à d'autres Roms (aver Roma, soit non Gabori), pour peu que l'on soupçonne une certaine proximité géographique ${ }^{20}$, la seconde ne peut s'adresser qu'à un semblable. Il s'agit en effet de situer l'autre dans les vastes réseaux de parenté qui structurent la nation gabor et, ainsi, de retrouver les divers liens qui unissent les interlocuteurs. Preuve sera alors faite qu'ils sont bel et bien semblables, membres d'un seul et même niamo.

41 Sémantiquement, le niamo romanès est en tout point comparable au neam roumain. Il correspond à l'idée large d'apparentement, pouvant signifier selon le contexte: " peuple », « famille », « lignage », etc. Le grand niamo que constitue ainsi la naŢia gabor est constitué de plusieurs niamuri de type "pseudo-lignages", les viŢi (viŢa au singulier).

Les viţi sont des groupes de descendance, généralement en ligne agnatique, portant le nom d'un ancêtre auquel s'ajoute le suffixe -eşti («ceux de ») : les Kuneşti sont les descendants de Kuna, les Djurkeşti ceux de Djurka, etc. À noter que cette pratique n'est en rien propre aux Roms. Neagu Djuvara explique comment, dans la paysannerie valaque d'autrefois (en Moldavie-Valachie comme chez les Roumains de Transylvanie), "le village entier se considérait comme descendant d'un ancêtre commun (un moş) et l'on gardait parfois par écrit la filiation des différentes lignées [...]. Le village portait d'ailleurs le plus souvent le nom de cet ancêtre éponyme, d'où la profusion de localités dont le nom se termine en -ești, suffixe pluriel désignant "les descendants de", "ceux de" $»^{21}$. L'expression neam de viţa renvoyait dans le parler roumain d'autrefois à l'idée de «bonne famille».

43 L'aïeul éponyme de la viŢa se situe à quatre ou cinq générations, rarement au-delà, la mémoire collective n'allant pas plus loin. Les vị̧i sont qualifiables de "pseudolignages ", car le système d'apparentement rom n'a rien de très formel et demeure éminemment malléable: chaque individu peut s'affilier à plusieurs viŢi selon les circonstances, celle de son père, de sa mère, parfois d'un grand-parent si celle-ci diffère... En effet, les mariages sont de préférence endogames à la viţa, mais l'exogamie est fréquente. C'est ainsi que tous les Gabori sont apparentés, toutes les viŢi possédant des liens d'alliance, mais qu'il y a toujours des parents qui le sont davantage : ceux de la même viŢa. Les membres d'une viŢa sont censés posséder certains traits de caractères communs, de même qu'ils partagent en héritage la renommée de leur aïeul : les Gabori sont tous «nobles" (rajkane) mais certains le sont plus que d'autres. Toute la parenté gabor se structure ainsi autour de ces jeux de valeur dans lesquels la généalogie est 
essentielle. Chaque viŢa établit son propre classement nobiliaire, plaçant les uns audessus des autres et se situant soi-même dans cette hiérarchie mouvante.

Chaque ensemble familial élargi (viţa ou portion de viţa) coïncide avec une géographie : à tel village correspond telle viŢa jugée particulièrement "noble ", tandis qu'à telle autre commune sont associées les images négatives d'un niamo peu respectable, de plus basse extraction. Il va sans dire que la hiérarchie des lieux, et donc des viŢi (ou viceversa), varie considérablement d'un interlocuteur à l'autre, selon sa propre position dans le champ des semblables. À l'heure actuelle, les Gabori sont néanmoins assez unanimes pour désigner la commune de Crăciunești, et en son sein le village de Budiu Mic, comme lieu d'origine de familles particulièrement nobles. C'est là que se situe la récente église adventiste gabor ${ }^{22}$.

Le nombre de communes et de villages gabor participant à cette logique de prestige fondée sur l'idée de noblesse héritée des ancêtres n'est pas indéfini. Si les Roms ne tomberont jamais d'accord sur la définition exacte de tous les lieux constituant leur terroir originel, il existe un consensus sur la majorité d'entre eux. On peut dès lors en établir la cartographie (fig. 2).

Figure 2. Département de Mureş et communes du terroir gabor (grisées)

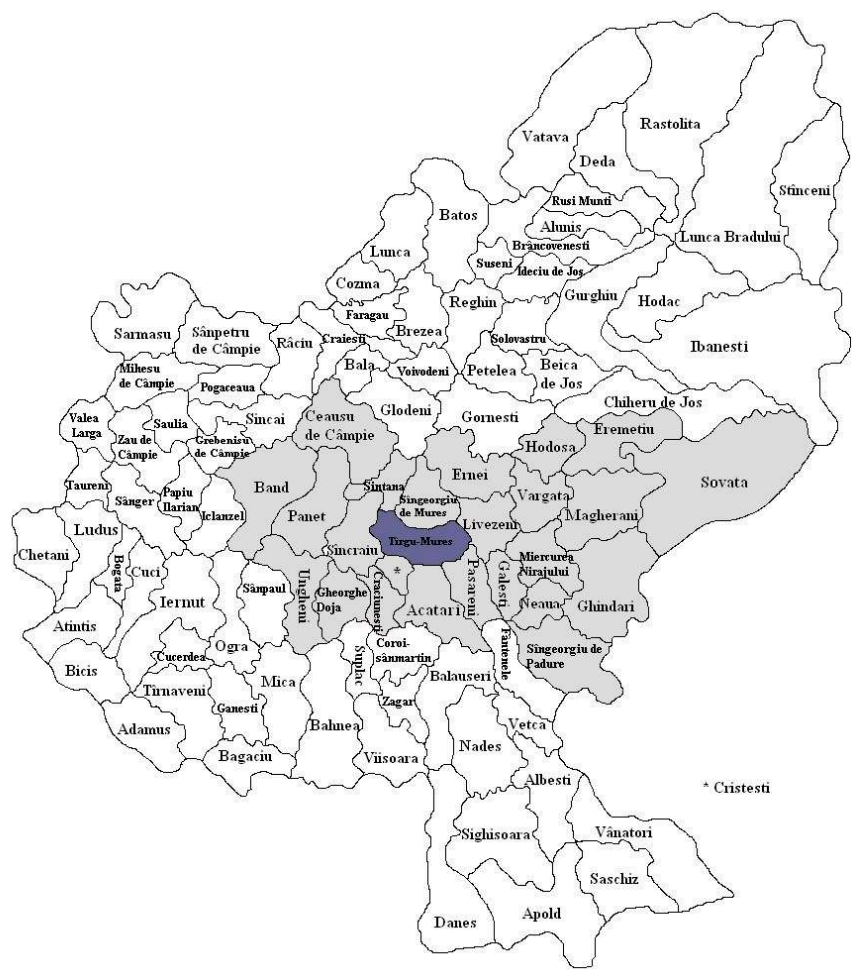

La carte présente l'actuel département de Mureş et, dans celui-ci, la quasi-totalité des communes d'où proviennent l'ensemble des viţi gabor ${ }^{23}$. Les familles roms y constituent en moyenne 5 à $10 \%$ de la population locale (selon le recensement de 2002). Sans former de quartiers à proprement parler, leur présence diffuse est donc non négligeable. À noter que les Gabori disposent d'une toponymie romanès propre (inspirée des noms hongrois) pour les communes de leur terroir, o foro (soit « la ville») désignant pour sa part Tîrgu-Mureş. On peut aisément constater qu'ainsi défini, le terroir gabor correspond presque exactement à l'ancien Siège de Mureş (Maros-szék), 
unité administrative disparue depuis la seconde moitié du XVIII ${ }^{\text {ème }}$ siècle. Le territoire sicule était en effet divisé jusqu'à cette époque en sept Sièges (fig. 3).

Figure 3. Principauté de Transylvanie sous le règnes des «trois nations " (XIVe-XVIIIe siècle) et Siège de Mureş (Maros)

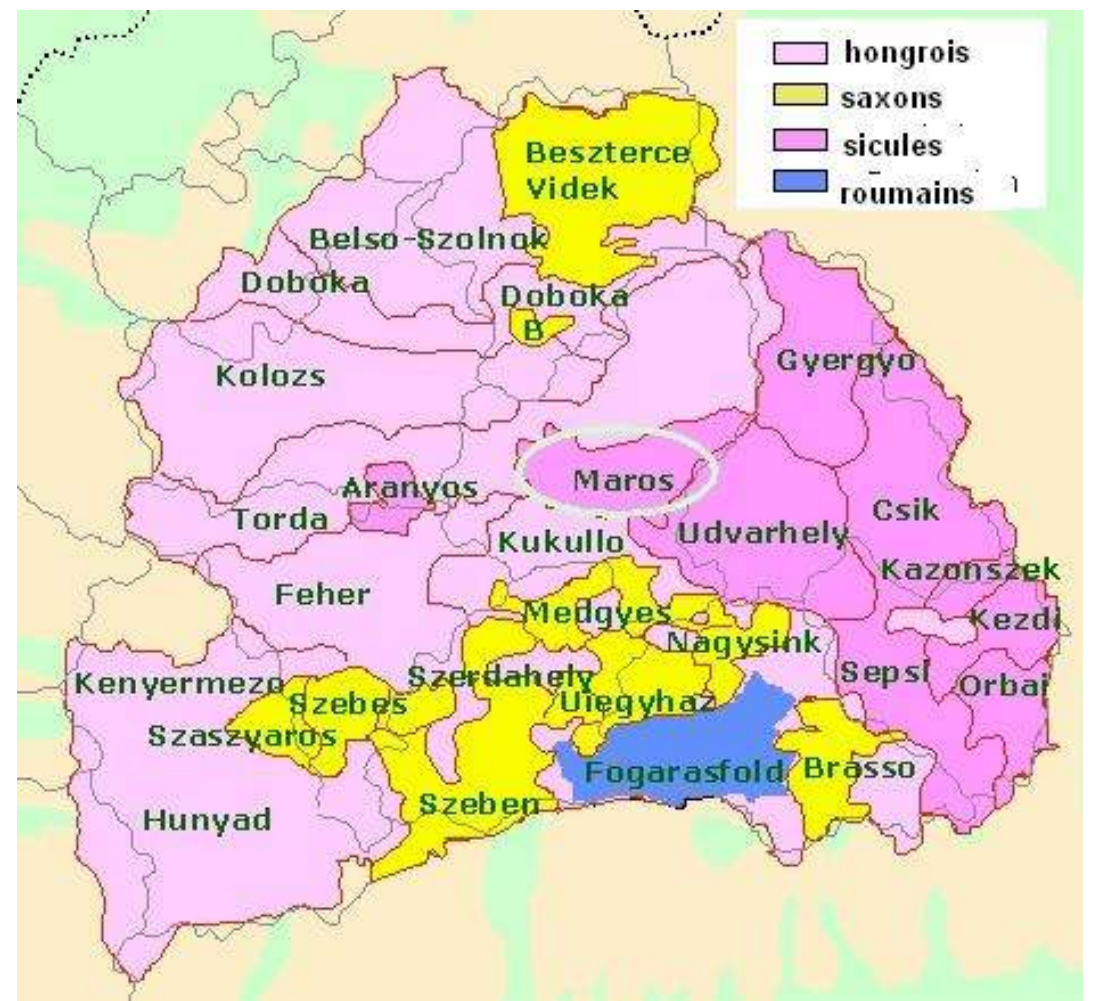
questionnements d'historiens n'ont, du point de vue des intéressés, aucun sens : ils ont toujours été là ${ }^{24}$. L'histoire, lorsqu'elle est établie par des éléments allogènes, est ennemie de l'autochtonie, la seconde ne pouvant s'établir que là où commence l'oubli. Car, Roms ou Gajé, les hommes ne naissent pas de la terre mais bien d'autres hommes. Et l'origine apparaît là où se perd l'ancestralité.

Voici ce que répond un vieux Rom à une enquêtrice sur la trace des authentiques Tsiganes nomades : «Seul le bon Dieu sait depuis combien d'années vit notre neam en pays sicule. Tous ensemble, avec nos familles, parcourant les villages, cherchant du travail, chaudronnerie, gouttières, rétamage de vaisselle. Mais à un moment, les familles se sont multipliées et l'on ne trouvait plus de travail. Alors notre "chef" a donné l'ordre de mettre enfants et bagages dans des carrioles et de partir de par le monde ! C'est comme ça qu'on est arrivés ici où il n'y avait pas d'autre famille installée. Tout cela c'est ma grand-mère qui me l'a raconté, mais elle disait qu'elle l'avait entendu de sa propre grand-mère comme quoi ça se serait passé quand son arrière-grand-mère était une fillette $»^{25}$.

L'installation gabor dans ce village remonterait ainsi à l'aïeule de l'aïeule d'un Rom aujourd'hui sexagénaire... Celui-ci précise par ailleurs dès le départ que de tous temps les Gabori ont été présents dans ce territoire particulier qu'est le pays sicule (secuime) : ils en parcouraient les villages puis, en raison de leur trop grand nombre, se seraient éparpillés... et installés dans ces mêmes villages qu'ils parcouraient déjà. L'informateur 
réussit par ce tour de force à inclure le «nomadisme », visiblement recherché par son interlocutrice, dans une autochtonie immémoriale.

Malgré l'éparpillement actuel des familles gabor, la référence au territoire originel et à ses villages est continuelle : qui veut identifier tel individu ou tel ensemble familial doit s'y reporter. L'organisation communautaire (fondée sur la parenté) perpétue ainsi au quotidien l'existence d'un terroir historique, fruit de la féodalité, qui ne représente plus rien pour l'immense majorité de leurs voisins Gajé. Le judet de Mureș actuel ne correspond en effet plus à l'ancien découpage en Sièges, et nombre d'habitants du département se disent de nos jours bien plutôt Hongrois que Sicules ${ }^{26}$. Ceux qui se jugent naturellement autochtones et regardent bien souvent les Tsiganes comme des étrangers, plus ou moins bien venus, ont perdu, sinon la mémoire, du moins la réalité sociale du « pays » de leurs aïeux, tandis que les Roms continuent de le faire vivre, d'en parler et d'en parcourir les collines et villages. Qui peut dès lors soutenir qu'ils ne sont «pas d'ici »?

1.

\section{Conclusion}

« D'où es-tu? ?, « De qui es-tu? » Ces questions récurrentes, les paysans roumains se les posaient également : «La coutume d'indiquer qu'un tel appartient à tel autre s'est maintenue dans le langage courant; les paysans roumains demandent encore avec qui est parent quelqu'un, pour mieux l'identifier. Lorsqu'on s'adresse à des enfants on dit : "comment t'appelles-tu ?" et ensuite "à qui appartiens-tu ?" (al cui eşti). »"27. Là encore, les Roms n'ont pas importé leurs coutumes d'un lointain ailleurs: l'ensemble des semblables correspondant aux membres de vastes réseaux de parenté interconnectés, eux-mêmes enracinés dans un territoire relativement restreint, équivalent du "pays " d'antan, le modèle est assez commun en Europe.

Les non-Tsiganes s'interrogent pourtant depuis plus de deux siècles sur l'« origine » des Tsiganes : d'où peuvent-ils bien venir puisqu'ils semblent si différents de ceux qui les entourent? L'exemple rapidement exposé ici, me semble démontrer deux choses.

En premier lieu que les intéressés, eux, savent très bien d'où ils sont: de tel village transylvain, de tel quartier de telle ville andalouse ${ }^{28}$, de telle province ou de tel canton français ${ }^{29}$, etc. Et leur autochtonie n'est pas moins réelle que celle des populations avec lesquelles ils vivent localement. Elle ne l'est pas davantage non plus : il y eut un temps où la société des Gabori n'existait pas, et il en sera un où elle n'existera pas. En attendant, ces Roms sont bel et bien d'ici et de maintenant, et non des allogènes hors du temps. Certes, l'autochtonie gabor semble davantage vécue que publiquement "fondée », au sens où l'entend M. Detienne ${ }^{30}$ : en dehors de celui livré aux Gajé (sur la respectabilité et la noblesse), il n'y a pas de discours interne objectivant l'identité gabor et ses fondements (mythe originel, héros fondateurs, lieux symboliques...). Tel est peutêtre ce "scandale » qui dérange tant les sociétés majoritaires. Mais ne pas tenir de discours sur les raisons de sa présence n'empêche pas d'habiter un lieu, et d'y être chez soi. pas les éléments de "morphologie sociale" ou les "traits culturels" qui sont propres mais le système auquel ceux-ci participent. Or ce système, le romanès (la manière des 
Roms d'être eux-mêmes), est tout entier le produit de l'intégration de chaque société rom à son environnement humain.

Cela n'est pas propre aux sociétés tsiganes, il se trouve simplement que la façon dont on les regarde (comme étant a priori allochtones) souligne ce fait essentiel : les sociétés humaines ne sont jamais seules et ne procèdent pas d'elles-mêmes. L'Autre a été et sera toujours là. Les Tsiganes ont ceci de commun qu'ils le savent intimement, d'expérience, quand les Gajé ne cessent de s'en étonner, pour le meilleur (l'anthropologie, entre autres), et pour le pire...

\section{NOTES}

Cf., par exemple, Thiesse (Anne-Marie), La création des identités nationales, Europe XVIIIe-XIXe siècle, Paris : Le Seuil, 1999 et Hobsbawm (Eric), Nations and Nationalism Since 1780: programme, myth, reality, Cambridge : Cambrige University Press, 1991.

Le Robert, dictionnaire historique de la langue française. Pour l'invention de la tsiganologie et les débuts de la « piste indienne » on rappellera notamment les travaux fondateurs des Orientalistes Johann Christian Christophe Rudiger (1777), Heinrich Grellman (1783) et, quelques décennies plus tard, le fameux Pott (August Friedrich), Die Zigeuner in Europe and Asien, 1844.

3. De ce point de vue, l'héritage social et politique revendiqué serait, d'ailleurs, plutôt exogène, cf. l'œuvre civilisatrice des Romains, des Francs et, plus récemment, des Américains, ....

4. Peu importe finalement le terme lorsqu'il veut englober la totalité de ces sociétés, puisqu'il ne désigne alors qu'une catégorie générique, objet des discours externes, et ne correspond pas aux réalités des groupes sociaux ainsi désignés.

5. Perspective largement remise en cause par l'anthropologie contemporaine, $c f$. par exemple Kuper (Adam), The invention of primitive society, Transformations of an illusion, London : Routledge, 1988 et Sahlins (Marshall), Des îles dans l'histoire, Paris : Gallimard / Le Seuil, 1989.

6. Hancock (Ian), Pariah Syndrome: An Account of Gypsy Slavery and Persecution, Ann Arbor : Karoma Publishers, 1987.

7. Cf. Okely (Judith), The Travellers-Gypsies, Cambridge: Cambridge University Press, 1983; Williams (Patrick), Mariage tsigane. Une cérémonie de fiançailles chez les Rom de Paris, Paris: L'Harmattan-Selaf, 1984 ; Piasere (Leonardo), Mare Roma. Catégories humaines et structure sociale. Une contribution à l'ethnologie tsigane, thèse de doctorat publiée par Études et documents balkaniques et méditerranéens (Paris), 8, 1985; Stewart (Michael), Brothers in Song. The Persistence of (Vlach) Gypsy Identity and Community in Socialist Hungary, Thesis, London : London School of Economic and Political Science, 1987 ; Reyniers (Alain), La Roue et la pierre. Contribution anthropologique à la connaissance de la production sociale et économique des Tsiganes, thèse de doctorat, Paris: Université de Paris V, 1992 ; Pasqualino (Caterina), Dire le chant, Les Gitans flamencos d'Andalousie, Paris : Éditions de la MSH, 1998.

8. De ce point de vue, le terme « Rom ", en romanès du moins, n'est pas à proprement parler un ethnonyme, $c f$. Olivera (Martin), « Les Roms comme "minorité ethnique" ? Un questionnement roumain », Études tsiganes (Paris), 39-40, 2009. 
9. Stewart (Michael), op.cit., p. 33. Pour de plus amples développements ethnographiques concernant les Gabori $c f$. Olivera (Martin), La tradition de l'intégration. Une ethnologie des Roms Gabori dans les années 2000, Paris : Pétra, 2012.

10. J'utilise «Transylvanie » dans son acception large, afin de désigner l'ensemble des provinces occidentales. Stricto sensu, la principauté historique de Transylvanie est toutefois à distinguer d'autres régions, administrativement séparées jusqu'au XIX ${ }^{\text {ème }}$ siècle : le Banat (départements actuels de Timiş et Caraş-Severin), la Crişana (Arad et Bihor), la région de Satu-Mare et le Maramureș.

11. Petit florilège transylvain : les habitants du département d'Alba sont censés être des bergers (ciobani), conduisant - forcément mal - des Dacia breaks fatiguées ; ceux de Cluj affectionneraient un accent précieux, expression d'un profond complexe de supériorité ; les Hongrois (Sicules) de Harghita et Covasna seraient particulièrement irascibles et prompts à la colère, etc. Bien entendu, tous ces stéréotypes sont valables en Transylvanie et demeurent étrangers aux habitants du sud valaque et autres Moldaves pour qui les ardelenii (transylvains) forment une entité homogène.

12. Houliat (Bernard), Tsiganes en Roumanie, Rodez : Éditions du Rouergue, 1999, p. 30

13. Cet usage n'est pas l'apanage des Gabori, d'autres Roms transylvains utilisent le terme pour désigner l'ensemble des semblables.

14. Tous les Hongrois, Saxons et Sicules n'étaient bien entendu pas nobles, mais de ceux-ci seulement émanait la noblesse. Les Valaques accédant à la classe dirigeante, et il y en eut tout au long de l'histoire de la principauté, se magyarisaient (cf. Köpeczi (Béla), éd., Histoire de la Transylvanie, Budapest : Akadémiai Kiadó, 1992). Le régime dit d'« union des trois nations » (Unio trium nationum) ne fut officiellement aboli qu'en 1848, sans jamais, toutefois, que les Valaques soient jamais reconnus comme « quatrième nation » transylvaine.

15. Dans les principautés de Moldavie et de Valachie, les dits Ţigani furent asservis dès le XIVème siècle (robie, souvent traduit par "esclavage »). Le terme Ţigan lui-même désignait ainsi une catégorie juridique plutôt qu'ethnique: les «serfs non autochtones", propriétés intégrales de leurs maîtres (autorités ecclésiastiques, boyards ou couronne princière). C'est au cours du XIX ${ }^{\text {ème }}$ siècle, alors qu'émerge le discours national «moderne" en Roumanie, notamment contre les privilèges féodaux et la servitude du petit peuple, que la figure du Ţigan s'impose dans la littérature puis dans les représentations populaires, comme vestige honnis de l'ère des boyards : l'idée apparaît, toujours valable aujourd'hui, que la Roumanie sera un pays «moderne» et « civilisé » lorsque, notamment, les Ţigani auront disparu en tant que Ţigani (par l'éducation et le développement dans le meilleur des cas - cf. Olivera (Martin), op.cit., pp. 83-105). À noter que les Roms eux-mêmes partagent ce point de vue, en parlant bien entendu des autres Tsiganes (ceux d'une autre région, ceux vus à la télévision, ceux des journaux, des contes populaires etc., bref, dans tous les cas des personnages fantasmés), non de leur propre communauté.

16. Les pionniers s'installèrent d'abord à Cluj et Oradea. Les suivants essaimèrent dans les années 1960-1970 dans toute la région, dans le Banat (Timişoara, Arad), puis en Hongrie et ailleurs en Roumanie.

17. Pons (Emmanuelle), Les Tsiganes de Roumanie, des citoyens à part entière ?, Paris : L'Harmattan, 1995.

18. Barth (Fredrik), Ethnic Groups and Boundaries. The Social Organization of Culture Difference, Oslo : Bergen, 1969.

19. Hobsbawm (Eric), Tanger (Terence), éds., The Invention of Tradition, Cambridge : Cambridge University Press, 1983.

20. Un Gabor ne demandera pas « d'où es-tu? » à un Rom qu'il a identifié comme étant originaire de Bucarest, ce ne serait d'aucun intérêt. En revanche, avec un autre Rom transylvain cela peut être pertinent : peut-être ont-ils des connaissances et amis communs dans telle ou telle ville, etc. 
Dans cette première phase d'interaction, l'accent (en romanès ou en roumain) est décisif pour identifier son interlocuteur.

21. Djuvara (Neagu), Le Pays roumain entre Orient et Occident. Les principautés danubiennes au début du XIXème siècle, Aurillac : Publications Orientalistes de France, 1989, p. 234.

22. Tous les Gabori ne sont pas évangélistes (pocaiţi en roumain, littéralement « repentis »), et parmi ces derniers tous ne sont pas Adventistes du Septième Jour: on trouve des Gabori baptistes, pentecôtistes, témoins de jéhovah... Actuellement, les groupes familiaux les plus renommés sont toutefois connus pour être adventistes.

23. Il faut y ajouter trois communes limitrophes appartenant aujourd'hui au département voisin de Harghita : Corund, Atid et Praid.

24. On peut noter dans le romanès gabor une forte influence hongroise, mais également roumaine. De ce point de vue, ils font partie de la catégorie linguistique des Roms dits "vlax" (valaques). A quelle époque leurs ancêtres auraient-ils franchi les Carpates en provenance des pays roumains (Moldavie-Valachie) pour s'installer en pays sicule? À ce jour, ce travail de recherche historique reste à mener.

25. Zriynyi (Irén), «Rromii Gabori » in G. Alexandrescu, éd., TraditŢii ale Rromilor din spaŢiul românesc, Bucarest : OrganizaŢia SalvaŢi Copii, 2004, p. 42.

Ma traduction. Dans le même recueil d'articles, un auteur évoque quelques pages plus loin les Gabori « à chapeaux [...] qui se sont installés depuis 310 ans dans les localités du département de Mureş ». D'où provient un tel chiffre ? L'auteur ne le dit pas ; constatons néanmoins qu'il s'agit de temps anciens.

26. Contrairement à leurs voisins de Harghita et Covasna qui, eux, tiennent encore à la distinction.

27. Stahl (Paul Henri), "Soi-même et les autres, quelques exemples balkaniques ", in LéviStrauss (Claude), éd., L'identité, Paris : PUF, 1977, p. 293.

28. Pasqualino (Caterina), op.cit.

29. Williams (Patrick), "Nous on n'en parle pas». Les vivants et les morts chez les Manouches, Paris : Éditions de la MSH, 1993 ; Reyniers (Alain), op.cit.

30. Detienne (Marcel), «L'art de fonder l'autochtonie, entre Thèbes, Athènes et le "français de souche" ", Vingtième siècle, Revue d'histoire (Paris), (69), janvier-mars 2001.

\section{RÉSUMÉS}

À partir de l'ethnographie des Roms Gabori de Transylvanie, cet article propose d'aborder les sociétés tsiganes comme des sociétés autochtones, à la fois produits et productrices de leur intégration locale fondamentale. À l'encontre de l'image de groupes sociaux hors du lieu et de l'histoire, ces Roms dits «traditionnels » donnent à voir le caractère profond, durable et actif de leur ancrage territorial et de leurs dynamiques culturelles.

From the ethnography of the Gabori Roma in Transylvania, this paper proposes to look at the Gypsy societies as indigenous societies, both products and producers of their fundamental local integration. Contrary to the image of social groups out of place and history, these "traditional" Roma demonstrate thedeep, durable and active character of their local anchorage and of their cultural dynamics. 
AUTEUR

MARTIN OLIVERA

Ethnologue, Docteur de l'Université Paris-10 Nanterre, membre du réseau scientifique européen Urba-rom

martin.olivera@yahoo.fr. 